\title{
Right open nephrectomy under combined spinal and peridural operative anesthesia and analgesia (CSE): A new anesthetic approach in abdominal surgery
}

\author{
Michele Cotugno $^{1}$, Matteo Dallaglio ${ }^{2}$, Luca Cantadori ${ }^{2}$, Fabio Villani ${ }^{2}$, Daniel Martens ${ }^{1}$, \\ Federico Cantoni ${ }^{1}$, Michele Potenzoni ${ }^{1}$, Salvatore Micali ${ }^{3}$, M.C. Bernardo Rocco ${ }^{3}$, Andrea Prati ${ }^{1}$ \\ ${ }^{1}$ Dipartimento Chirurgico, U.O. di Urologia, Ospedale di Vaio-Fidenza, Fidenza, Italy; \\ ${ }^{2}$ Dipartimento Chirurgico, U.O. di Anestesia e Rianimazione, Ospedale di Vaio-Fidenza, Fidenza, Italy; \\ ${ }^{3}$ Dipartimento di Chirurgia Generale e Specialità Chirurgiche, U.O. di Urologia, Azienda Ospedaliero-Universitaria di Modena, Italy.
}

\begin{abstract}
Summary
A case of right open nephrectomy performed under combined spinal and epidural anes-

thesia and analgesia was presented.

This new anesthetic technique gives significant advantages to the patient by avoiding endotracheal intubation with mechanical ventilation and curare administration and by reducing the use of opioids.
\end{abstract}

KEY WORDS: Nephrectomy; Spinal; Epidural; Anesthesia.

Submitted 5 June 2019; Accepted 2 September 2019

\section{INTRODUCTION}

We describe a case of right open nephrectomy with xifopubic incision in a patient undergoing initially thoracic spinal anesthesia that was later converted into peridural anesthesia and analgesia (CSE).

\section{Patients And methods}

A 57-year-old male patient with negative medical history for major internal and surgical diseases accessed our operative unit for a massive hematuria. Abdomen ultrasound showed a right renal mass suspected as heteroplasia. Subsequently he performed a computed tomography (CT) of the abdomen with evidence of an inhomogeneous and hyper-vascularized renal neoformation of $10 \times 9 \mathrm{~cm}$ with central necrotic area and without safe cleavage from the liver. Presence of clots in the renal pelvis, numerous mesenteric and retroperitoneal adenopathies and an occasional finding of a thoracic aortic aneurysm of $4.8 \mathrm{~cm}$ were also demonstrated (Figures 1,2). It was decided to perform the intervention of right nephrectomy with xifopubic incision given the size of the mass and the unsafe cleavage from neighboring structures.

Anesthesiologist performed a Combined Spinal and Epidural (CSE) anesthesia and analgesia: subarachnoid puncture at T10-T11 level with Whiteacre 25G needle with administration of isobaric bupivacaine $0.5 \% 10 \mathrm{mg}$ + dexamethasone $4 \mathrm{mg}$ + ketamine $20 \mathrm{mg}$ + midazolam $2 \mathrm{mg}$ reaching a complete sensory and motor block up to T3 level. Immediately after the spinal puncture, a peridural catheter was placed at T9-T10 level and the right radial artery was cannulated. Beginning of the operation was about 15 minutes after the puncture without any surgical or anesthetic problem. After about 70 minutes $15 \mathrm{ml}$ of ropivacaine $0.5 \%$ was administered in the peridural catheter in fractions of $5 \mathrm{ml}$ each in a total time of 30 minutes.

In the meantime, peridural infusion of ketamine $50 \mathrm{mg}$ + midazolam $5 \mathrm{mg}$ was started at a rate of $2-3 \mathrm{ml} / \mathrm{h}$.

The patient was sedated in a light way, awake to the call and reactive to the light stimulus. He maintained spon-

Figure 1.

Antegrade pyelography.

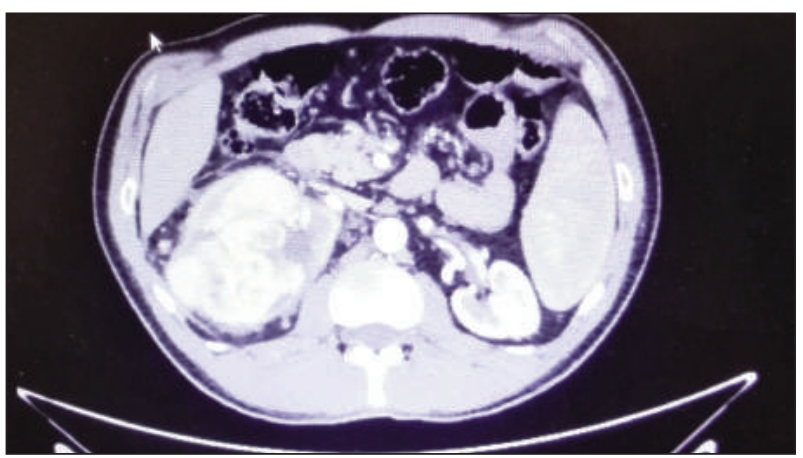

Figure 2.

Extraperitoneal approach.

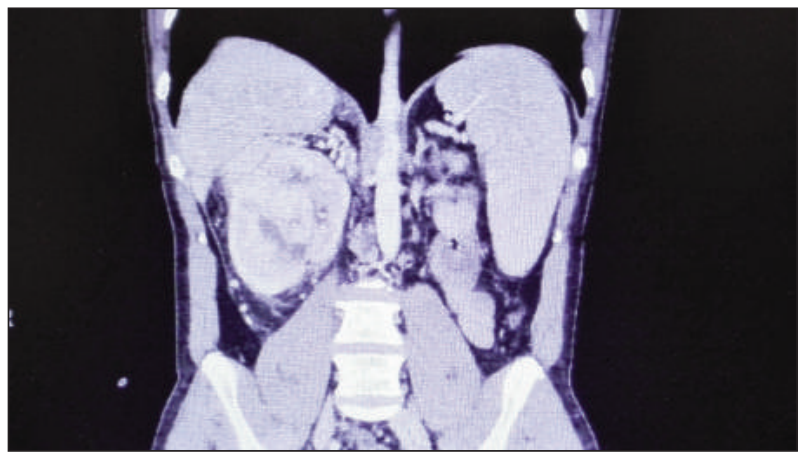


taneous breathing with nasal cannulas and low oxygen flow achieving excellent saturation values. The intervention lasted a total of $2 \mathrm{~h}$ and 40 minutes with blood losses of about $200 \mathrm{ml}$.

Starting from the closure of the surgical wound, ropivacaine $0.2 \%$ infusion was initiated in association with midazolam $5 \mathrm{mg}$ at $5 \mathrm{ml} / \mathrm{h}$. Patient controlled analgesia (PCA) was continued with $1.5 \mathrm{ml}$ boluses with lockout every 20 minutes.

\section{RESULTS}

Arterial Blood Gas (ABG) at the end of the procedure showed excellent respiratory exchanges with normocapnia, and lactate in the normal range.

Post-operative course was regular. Vital parameters always remained in the normal range, gastrointestinal canalization was recovered the day after the operation, blood count was stable. Bladder catheter was removed on the third postoperative day. Subhepatic drainage and epidural catheter were removed on the fourth postoperative day. The apyretic and asymptomatic patient was discharged on the seventh postoperative day waiting for result of histological examination.

\section{Discussion}

Castellani et al. were the first in Italy to describe five cases of radical cystectomy performed under continuous spinal anesthesia, a technique that represents the natural evolution of that used by us (1). Numerous authors have shown excellent perioperative outcomes in cardio-vascular, orthopedic, pelvic and abdominal surgery (2-4).

It is also interesting to note that the entire procedure was conducted in opioid free mode without negative effect on pain during intra- and post-operative period. This is the first case described in Italy of open nephrectomy performed with this type of anesthesia and the excellent results obtained leads us to think that we can apply it in other future surgical procedures.

\section{Conclusions}

Our opinion is that this new anesthetic technique gives significant advantages to the patient, in particular by avoiding endotracheal intubation with mechanical ventilation and curare administration and by reducing the use of opioids. The evolution (using a special device) to continuous spinal anesthesia (CSA) could lead to a further improvement of the procedure. Randomized and controlled clinical trials will be needed to confirm our initial results.

\section{REFERENCES}

1. Castellani D, Starnari R, Faloia L, et al. Radical cystectomy in frail octogenarians in thoracic continuous spinal anesthesia and analgesia: a pilot study. Ther Adv Urol. 2018; 10:343-349.

2. Michaloudis D, Petrou A, Bakos P, et al. Continuous spinal anaesthesialanalgesia for the perioperative management of high-risk patients. Eur J Anaesthesiol. 2000; 17:239-247.

3. Amin SM, and Sadek SF. Continuous spinal anesthesia for elderly patients with cardiomyopathy undergoing lower abdominal surgeries. Egypt J Anaesth. 2016; 32:535-540.

4. Jaitly VK and Kumar CM. Continuous spinal anaesthesia for laparotomy. Curr Anaesth Crit Care. 2009; 20:60-64.

\section{Correspondence}

Michele Cotugno, MD (Corresponding Author)

mikcot88@libero.it

Daniel Martens

dmastens@ausl.pr.it

Federico Cantoni

fcantoni@ausl.pr.it

Michele Potenzoni

mpotenzoni@ausl.pr.it

Andrea Prati

aprati@ausl.pr.it

Dipartimento Chirurgico, U.O. di Urologia Ospedale di Vaio-Fidenza,

Fidenza (Italy)

Matteo Dallaglio

madallaglio@ausl.pr.it

Luca Cantadori

lcantadori@ausl.pr.it

Fabio Villani

fvillani@ausl.pr.it

Dipartimento Chirurgico, U.O. di Anestesia e Rianimazione,

Ospedale di Vaio-Fidenza, Fidenza (Italy)

Salvatore Micali

smicali@unimore.it

Maria Cesare Bernardo Rocco

bernardo.rocco@gmail.com

Dipartimento di Chirurgia Generale e Specialità Chirurgiche, U.O. di Urologia,

Azienda Ospedaliero-Universitaria di Modena, Modena (Italy) 